experience, were eligible. From those, 741 participants completed at least one of the health monitors and were included in the analysis.

Intervention The Runfitcheck is an online intervention to stimulate injury-preventive behavior. The participants were allocated to one of two intervention groups or the control group. One intervention group obtained access to the Runfitcheck and was fortnightly stimulated to use Runfitcheck, the other intervention group was attended to the Runfitcheck once. Participants were followed over a period of four months.

Main outcome measures The main outcome measure was a new RRI, measured fortnightly with the Oslo Sport Trauma Research Centre overuse injury questionnaire.

Results The time to the occurrence of the first RRI did not differ between the study groups (Wald Chi-square 0.893). There was also no difference in risk of a new RRI in the group attended to the Runfitcheck once (OR $1.22 \quad(95 \%$ CI:0.86-1.74)) nor in the active approach group (OR 1.01 (95\%CI: 0.71-1.45)) compared to the control group. Furthermore, the onset of the new RRIs did not change over time (OR 0.96 (95\%CI: 0.91-1.01)).

Conclusions The online intervention Runfitcheck was not effective in reducing the risk of new RRIs in adult novice runners. More research is needed to determine how injuries in novice runners can be prevented.

\section{DEVELOPMENT OF A PROTOCOL TO EVALUATE BASEBALL PITCHER'S WORKLOAD AND PREVENTION OF INJURY}

Jason L Zaremski, Pazik N Marrisa, Horodyski MaryBeth. University of Florida, Gainesville, Florida, USA

\subsection{6/bjsports-2021-IOC.196}

Background Pitch counts are one measure of workload in baseball. Newer research indicates pitch counts underestimate true total workload. Thus, current monitoring systems gauging throwing injury threshold may be considered inadequate.

Objective Develop a novel technique to determine workload in baseball pitchers to provide an implementable method for prevention of throwing related injuries.

Design Prospective observational study

Setting Academic medical center and community baseball fields.

Participants Pitchers aged 13-18 from the 2019 to 2021 seasons.

Assessment of Risk Factors The independent variable was innings pitched, grouped by 1-2 innings, 3-4 innings, 5-6 innings, and 7 innings.

Main Outcome Measurements Workload percent, calculated by multiplying volume of total gameday pitches by intensity, was the primary measure. Intensity was determined by maximum pre-season velocity compared to game velocity of pitches thrown. Velocity was measured during a preseason practice and the first 10 pitches of each inning. Total gameday pitches included bullpen, warm-up, and game pitches.

Results 147 total pitcher outings, 42 total pitchers, 4 pitching related injuries. Total game pitch counts ranged from 17 to 219 (mean 78.8, SD 38.7). Velocity ranged from 74.4 to
$136.5 \mathrm{~km} /$ hour. Intensity ranged from 0.68 to 1.26 with a mean of 1.0 (SD 0.09). ANOVA was completed with significant differences noted for total pitches $(p<0.001)$ and workload percent $(\mathrm{p}<0.001)$. Post hoc analyses for total pitches and workload percent resulted in significant differences $(\mathrm{p}<0.001)$ between all inning groupings except innings 5-6 compared to inning 7.

Conclusions Our workload model indicated that workload and pitch counts are associated. As we gather more data (2022 season), if workload and injuries are significantly associated, then by extension total gameday pitch count would become a correlate to workload and injuries. Workload monitoring using our system may lead to prevention of injuries in baseball pitchers.

\section{SPORTS INJURY PREVENTION PRACTICES AND DIRECTIONS FOR IMPROVEMENT: A MULTI-CENTRE QUALITATIVE STUDY IN PHYSICAL EDUCATION TECHER EDUCATION STUDENTS}

${ }^{1}$ Sander Bliekendaal, ${ }^{2}$ Maarten Barendrecht, ${ }^{3}$ Janine Stubbe, ${ }^{1}$ Caroline Bolling, ${ }^{1}$ Evert Verhagen. ${ }^{\prime}$ Amsterdam Collaboration on Health and Safety in Sports, Department of Public and Occupational Health, Amsterdam Movement Science, Amsterdam UMC, Amsterdam, Netherlands; ${ }^{2}$ Mijn Fysio en Adviespunt, Den Haag, Netherlands; ${ }^{3}$ Codarts University of the Arts, Rotterdam, Netherlands

\subsection{6/bjsports-2021-IOC.197}

Background Injury risk is high in Physical Education Teacher Education (PETE) student. Insight in the population's perspectives on injury prevention supports developing context-driven preventive strategies.

Objective The objective was to describe sports injury prevention practices from PETE students' perspective and map their suggestions for improvement.

Design Qualitative study.

Setting PETE programs at Dutch Universities of Applied Sciences.

Participants Recruitment took place via convenience sampling. Students were invited to participate by e-mail. Participants were informed about the study's goals and procedures and completed informed consent and a baseline questionnaire (e.g., age, sports participation, injury history). Inclusion criteria for participants were: 1) completed the first three years of the PETE program; 2) understanding of the Dutch language at the native level. In total 21 participants from four different PETE schools were included.

Main Outcome Measurements We conducted semi-structured interviews, which were transcribed verbatim and analysed using the thematic analysis method.

Results All the participants mentioned applying various preventive measures. They described injury prevention as a standard part of daily life an approached it in a dynamic way. Their primary strategy was to balance load and recovery. The critical factors to apply injury prevention successfully mentioned were: communication, learning what works, self-management, shared responsibilities, and social support. The main motives for injury prevention were to care for the body and perform well (e.g., academic success, sports). Given the participants, injury prevention could be improved in various ways, but mostly by enhancing the PETE program's load management 